\title{
Treatment of anterior canal benign paroxysmal positional vertigo by a prolonged forced position procedure
}

\section{Crevits}

This report presents a therapeutic procedure for refractory benign paroxysmal positional vertigo (antBPPV) of the anterior canal. Two patients with refractory antBPPV were treated by a prolonged forced position procedure (PFPP). The technique is based on the assumption that the pathophysiological mechanism of antBPPV is similar to that generating posterior canal canalolithiasis. The patients recovered from refractory antBPPV after one or more PFPPs. The rationale for this therapy is that when the patient lies in the proposed forced position, the affected anterior canal is uppermost in an almost gravitationally vertical position. If the patient remains in this position for several hours, the floating particles lying in the non-ampullary arm of the canal can gradually slip out of the canal towards the vestibule due to gravity. We recommend trying PFPP when the side of lithiasis cannot be determined, in cases that are resistant to particle repositioning canal manoeuvres, and before considering canal plugging for refractory antBPPV.

B enign paroxysmal positional vertigo (BPPV) usually presents as a distinct clinical syndrome characterised by brief spells of violent rotational vertigo induced by changes in head position. The diagnosis is confirmed by typical findings of the Dix-Hallpike positioning tests. ${ }^{12}$ BPPV can involve any of the semicircular canals (SC), although the posterior SC type is by far the most frequent. BPPV commonly is attributed to either canalolithiasis or cupulolithiasis (debris free floating in the canal or attached to the cupula). ${ }^{1}$ Although remission is common and the condition is benign, persisting episodic BPPV may be disruptive for patients. Therapy is mechanical and aimed at moving the free floating debris into the vestibule. However, the regimen must be specific to type and location of the lithiasis.

The anterior SC BPPV (antBPPV) is not frequent. Honrubia et al described four patients (1.3\%) with anterior SC canalolithiasis out of 292 patients with BPPV. ${ }^{2}$ The initial evaluation of this group provides a clear indication of the relative involvement of the anterior SC. An incidence of less than $2 \%$ of BPPV cases was confirmed in a retrospective series. ${ }^{3}$ Anterior SC canalolithiasis is assumed to be rare due to its anatomically superior position during most activities and the posterior arm of the anterior SC descending directly into the common crus and vestibule. Thus, debris within the anterior canal should be self clearing. It is unclear why this does not happen in antBPPV. The almost vertical orientation of the ampullary segment of the anterior SC could result in extensive contact between the debris and the cupula, thus facilitating cupulolithiasis. In contrast to canalolithiasis, cupulolithiasis does not show adaptation and habituation of vertigo and nystagmus. ${ }^{2}$

In anterior SC canalolithiasis, the nystagmus is mainly down beating with a smaller torsional component. The torsional component may be weak because of the predominantly sagittal orientation of the anterior canal, and may not be readily seen clinically. Video-oculography may be necessary for its detection. Moreover, the direction of the torsional component does not seem to be uniform or is disputed.$^{3-5}$ It is striking that the Dix-Hallpike test could provoke a nystagmus from either side. This can be explained by the upward vertical orientation of the ampullary segment of the anterior SC in the normal upright head position. This orientation requires a further downward movement of the head, often achieved with the straight head-hanging position, to provoke migration of the canaliths. ${ }^{5}$

Therapeutic information for antBPPV is extremely scarce. Honrubia et al mention a "reverse Epley" particle repositioning procedure (PRP) and obtained success in two of four patients. ${ }^{2}$ No further details of the manoeuvre were given. Rahko studied a remarkably large group ( 57 patients) that had residual symptoms of antBPPV after treatment for posterior and horizontal BPPV. ${ }^{6}$ He applied a treatment manoeuvre whereby the patient lies on the healthy side, the head is tilted downwards $45^{\circ}$, then horizontally, then upwards $45^{\circ}$ for 30 seconds each, and finally the patient sits up and stays there, well supported, for at least three minutes. "Good results" were ascribed to the fact that the other canals had been treated before. However, the diagnosis and results were not based on nystagmus characteristics and relied on methods that have not been validated.

We introduce a prolonged forced position procedure (PFPP) as treatment for refractory antBPPV.

\section{METHODS \\ Patients}

Case 1

A 50 year old woman complained of episodic vertigo lasting several hours and occurring once a month since four years. The vertigo was accompanied by tinnitus on the left side. She was prescribed betahistine.

She presented to the otoneurological unit because of a new complaint of short lasting paroxysmal positional vertigo since several weeks. The positional vertigo was brief (seconds), consisting of a sense of spinning and manifesting mainly on lying down. Prior history included hypertension and migraine. There was no history of trauma.

The right Dix-Hallpike manoeuvre triggered positional vertigo and a down beating nystagmus (DN) - that is, fast phase beating to the chin. The DN had a latency of four seconds, a duration of 15 seconds and habituated on repeated testing. No torsional component could be detected under Frenzel's glasses. In the left Dix-Hallpike and in the head-hanging position, a similar DN pattern could be elicited,

Abbreviations: AntBPPV, anterior SC BPPV; BPPV, benign paroxysmal positional vertigo; DN, down beating nystagmus; MRI, magnetic resonance imaging; PFPP, prolonged forced position procedure; PRP, particle repositioning procedure; SC, semicircular canal 
but with a lesser intensity, and reversal of the nystagmus did not occur. Horizontal SC manoeuvres were negative. Neurological examination was normal.

Caloric and rotatory responses showed a directional preponderance of the induced nystagmus to the right. Pure tone audiogram, electrocochleogram and brainstem auditory evoked potentials were normal. A brain magnetic resonance imaging (MRI) scan was unremarkable.

The longer lasting episodic vertigo with tinnitus on the left and the vestibular findings suggested left labyrinthine involvement. The positional tests were compatible with antBPPV.

Because the side of the antBPPV could not be determined, we tried a PFPP (see below) instead of a reverse Epley PRP. Two days after the PFPP, the patient was re-examined and no nystagmus could be elicited on positional tests. She remained free of vertigo for three months. Then she again experienced very short bursts of positional vertigo. In the right DixHallpike, a DN of duration three seconds was seen after a latency of 30 seconds. The complaints, however, were not considered severe enough to be treated. However, after five months, the vertigo became more embarrassing. On examination, the right Dix-Hallpike manoeuvre elicited a transient DN and a torsional component was observed. The direction, however, could not be specified under Frenzel's glasses. A reverse Epley PRP remained unsuccessful after two trials. A second PFPP was successful. No positional vertigo or nystagmus could be elicited immediately after the PFPP and during a follow up period of three months. Then the patient had a new episode of antBPPV. Neurological examination remained normal and a repeat MRI of the brain and inner ear did not reveal any anatomical anomalies, especially the anterior SC showed normal morphology on both sides. The patient was treated with a PFPP for the third time.

\section{Case 2}

This 57 year old woman was known to have Ménière's disease since 13 years and was being treated with betahistine. The history included a skull fracture of the left occipital bone in a traffic accident 36 years ago, one tonic clonic seizure four years ago that had been treated with carbamazepine for some months, and several hyperventilation attacks. She presented because of positional vertigo when turning over in bed especially to the right side, since two months.

The right and left Dix-Hallpike manoeuvres, as well as positioning to head-hanging evoked positional vertigo and a DN (fast phase beating to the chin). The pattern of the DN was essentially similar in the three positions. Latency was of 3-5 seconds, duration 5-15 seconds and habituation occurred on repeated testing. In the left Dix-Hallpike position, a discrete torsional (geotropic) component was suspected and reversal of the nystagmus did not occur. Horizontal canal manoeuvres were negative. Neurological examination was unremarkable.

Vestibular tests revealed caloric hypofunction on the right. Pure tone audiometry showed a right neurosensorial hearing loss of $45 \mathrm{db}$, nearly flat for frequencies from $250 \mathrm{~Hz}$ to $8000 \mathrm{~Hz}$. Electrocochleography was disturbed on the right for tones and clicks. Electroencephalography, MRI of the posterior fossa and $x$ rays of the craniocervical junction were negative.

The patient was admitted for a PFPP (see below). However, the procedure had to be terminated prematurely due to neck pain. Because of refractory positional vertigo for another five months, the PFPP was tried again, but now with myorelaxation using diazepam. The patient was re-examined immediately after the PFPP and twice during a follow up period of seven months. No positional nystagmus could be provoked and the patient remained free of vertigo, except for one typical Ménière attack.

\section{Prolonged forced position procedure}

For the PFPP, the patients were admitted to hospital. In an orthopaedic bed, the positional test with hanging head was first carried out to induce DN and to be sure of the provoking position. Thus the patient was rapidly moved from sitting to lying supine with the head bent backwards as far as possible-that is, with the vertex about $60^{\circ}$ below the horizontal. The head was supported in this hanging position for 30 minutes. During this period, the debris were thought to sediment into the superior part of the anterior canal (now the most dependent) and clot (fig 1). Then the head was moved quickly forwards, as far as possible-that is, with the vertex near the vertical. Due to inertia, the canaliths lag somewhat behind and then move to the common crus of the anterior and the posterior canals (fig 1). In this forced position, the head was fastened with the help of a pulley system that allowed the head to hang slightly (fig 2). The patient was not allowed to stand up. After 24 hours, the patient was asked to sit and stay in the sitting position with the head straight for some minutes to allow the clot to move further in the utricle through the common crus. Following this no position or movement restrictions were applied. After the PFPP, positional tests were repeated to verify the effect and to exclude conversion into another variant of BPPV.

\section{DISCUSSION}

Central positional nystagmus can be mistaken for BPPV. This is especially true for DN, being posture dependent and known to have pluriform manifestation. ${ }^{78} \mathrm{DN}$ can be caused either by cerebellar degeneration, Arnold-Chiari malformation and other dysfunctions of the cerebellar flocculus, or by lesions of the medial longitudinal fasciculus in the floor of the fourth ventricle. ${ }^{9}$ Recently, Bertholon et al considered possible anterior SC canalolithiasis in patients with positional DN without evidence of central nervous dysfunction. ${ }^{5}$ One problem of antBPPV concerns the contradictory interpretations as to whether an ipsilesional or a contralesional Hallpike manoeuvre provokes nystagmus. Due to the coplanar orientation of the left posterior canal with the right anterior canal, the left Hallpike manoeuvre should provoke a right antBPPV. ${ }^{10}$ However, a left-right lateralisation seems less specific in antBPPV as in the other canal types. ${ }^{511}$ It has been suggested that the anterior SC can be cleared of canaliths by using the same positioning sequence for contralateral posterior canalolithiasis. ${ }^{12}$

The present patients suffered from longstanding vestibulopathy of the Ménière type. ${ }^{13}$ However, they presented with paroxysmal positional vertigo and manifested positional DN

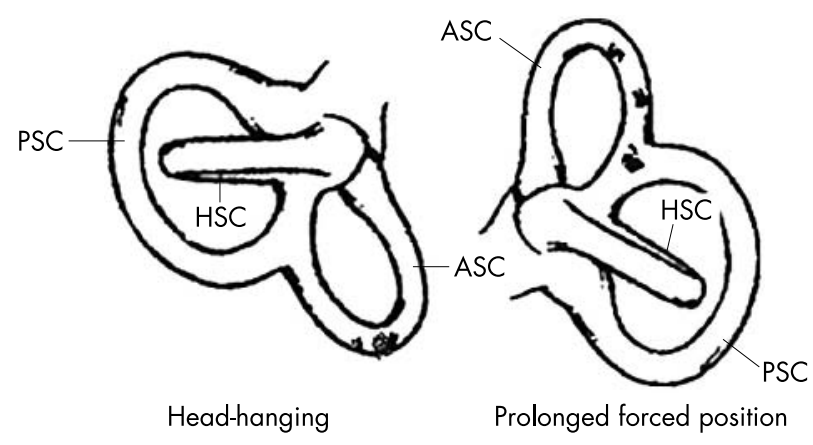

Figure 1 Schematic representation (lateral view) of the semicircular canals with debris in the uppermost part of the anterior canal in the head-hanging position. In the prolonged forced position for anterior canal benign paroxysmal positional vertigo, the debris moves into the common crus and the vestibule. ASC, PSC, HSC, anterior, posterior and horizontal semicircular canal, respectively. 


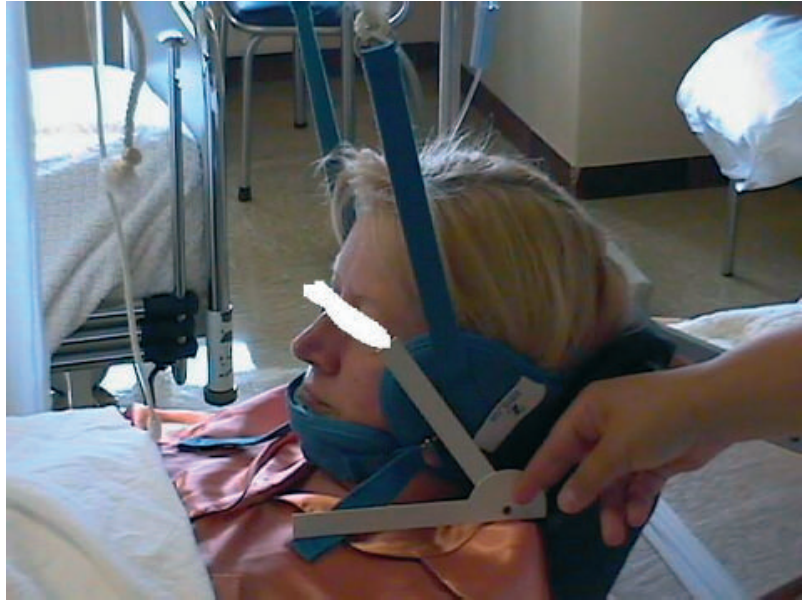

Figure 2 A patient in the prolonged forced position for anterior canal benign paroxysmal positional vertigo. The arms of the goniometer indicate the angle between the vertex and horizontal in the headhanging position. From the head-hanging position the head has been moved forwards over about $120^{\circ}$ to achieve the position shown.

with features of BPPV (latency, short duration and habituation or fatigability of the nystagmus). There were no signs of neurological disorders, especially multiple system atrophy, cerebellar degeneration, and Arnold-Chiari malformation could be ruled out. As our patients had not been treated before, their antBPPV could not be ascribed to a complication of a PRP. ${ }^{4}$ Since the site of the lesion could not be determined the reverse Epley manoeuvre or the procedure of Rahko could not be performed although the reverse Epley PRP was tried without success in case 1 .

The reason why anterior SC canalolithiais continues for a long time or relapses (case 1) could be due to the conglomerate of debris being too large, continuous production of debris, failure of disaggregating, or narrow common crus. It is to be noted that in our patients antBPPV might have been influenced by concomitant peripheral vestibulopathy. However, this could be coincidental. Cupulolithiasis can be a cause of refractory BPPV; however, in the case of cupulolithiasis less adaptation or habituation should occur. Canalith "jam" is another type of vestibular lithiasis. It refers to locking of the cupula in a fixed position by debris, as can occur as a complication of a PRP. ${ }^{2}{ }^{12}$ The ensuing vertigo and nystagmus, however, are not affected by head position. Hence the reason for refractory antBPPV in the present patients remained unclear.

We applied a new therapeutic procedure for antBPPV. Our technique is based on the assumption that antBPPV has a pathophysiological mechanism similar to the one generating posterior SC canalolithiasis-that is, there are some particles heavier than endolymph that are free to move into the SC and that are capable of causing some abnormal endolymphatic movement due to the force of gravity. Freeing the canal of all its particles can prevent this process. This concept led us to suggest that therapy for antBPPV would be effective when the patient lies with the head fixed upward for a long time. This forced prolonged position might allow the heavy particles to gradually be drawn into the vestibule by the force of gravity. Prior to the forced position, however, the debris has to sediment in the superior part of the anterior SC with the patient lying down in the head-hanging position as described in the Methods section.

Since spontaneous remission of BPPV is common, improvement cannot be clearly ascribed to therapy. However, our patients had, and ultimately recovered from, refractory antBPPV. The time course too, was very suggestive of a relation between PFPP and remission of antBPPV. The best way to know whether the patients are symptom free because of the manoeuvre or as a result of spontaneous recovery is to examine the patients the same day, after a sufficient interval to avoid fatigability effects. ${ }^{14}$ Therefore, patients were examined immediately after the PFPP and repeatedly during the ambulatory follow up to verify recovery and exclude conversion into another variant of BPPV.

The PFPP presented here has the advantage over PRP of circumventing the difficult problem of lateralisation of antBPPV. The rather long and uncomfortable bed stay for 24 hours is an inconvenience. The technique can have complications: if the head is brought too far forwards during the PFPP, the debris could move to the ampullar part of the anterior SC causing cupulolithiasis. Therefore, the pulley system should allow the head to hang slightly backwards. When the head bends too far backwards, the clot can descend into the posterior SC. Fortunately, this complication can easily be detected by a Dix-Hallpike positioning test and be cured by an Epley PRP. Conversion into another variant of BPPV can not always be avoided due to individual anatomical variation in orientation of the SC. Therefore, a careful examination after the PFPP is mandatory. A side effect of keeping the head in the forced position is a stiff neck and therefore myorelaxation is indicated. We recommend trying PFPP when the side of lithiasis cannot be determined, in cases that are resistant to particle repositioning canal manoeuvres, and before considering canal plugging in refractory cases of antBPPV.

Competing interests: none declared

Correspondence to: Luc Crevits, MD, PhD, Department of Neurology, Oto-Neuro-Ophthalmology Unit, Ghent University Hospital, De Pintelaan 185, B-9000 Ghent, Belgium, Tel: +32.9.240.45.39, Fax: +32.9.240.49.71; E-mail: luc.crevits@ugent.be

Received 7 August 2003

In revised form 12 October 2003

Accepted 22 October 2003

\section{REFERENCES}

1 Bronstein AM. Benign paroxysmal positional vertigo: some recent advances. Curr Opin Neurol 2003;16:1-3.

2 Honrubia V, Baloh RW, Harris MR, et al. Paroxysmal positional vertigo syndrome. Am J Otol 1999;20:465-70.

3 Korres S, Balatsouras DG, Kaberos A, et al. Occurrence of semicircular canal involvement in benign paroxysmal positional vertigo. Otol Neurotol 2002;23:926-32

4 Herdman S, Tusa RJ. Complications of the canalith repositioning procedure. Arch Otolaryngol Head Surg 1996; 122:281-6.

5 Bertholon P, Bronstein AM, Davies RA, et al. Positional downbeating nystagmus in 50 patients: cerebellar disorders and possible anterior semicircular canalithiasis. J Neurol Neurosurg Psychiatry 2002;72:366-72.

6 Rahko T. The test and treatment methods of benign paroxysmal positional vertigo and an addition of the management of vertigo due to the superior vestibular canal (BPPV-SC). Clin Otolaryngol 2002;27:392-5.

7 Crevits L. Downbeat nystagmus. Pluriform manifestation. Neuroophthalmology 1991;11:103-4.

8 Crevits L, Reynaert C. Posture dependent direction reversal of spontaneous vertical nystagmus. Neuro-ophthalmology 1991;11:285-7.

9 Baloh RW, Spooner JW. Downbeat nystagmus: a type of central vestibular nystagmus. Neurology 1981;31:304-10.

10 Bronstein AM. Vestibular reflexes and positional manoeuvres. J Neurol Neurosurg Psychiatry 2003;74:289-93.

11 Brantberg K, Bergenius J. Treatment of anterior benign paroxysmal positional vertigo by canal plugging: a case report. Acta Otolaryngol 2002;122:28-30.

12 Epley JM. Human experience with canalith repositioning maneuvers. NY Acad Sci 2001;942:179-91.

13 American Academy of Otolaryngology, Committee on Hearing and Equilibrium. Guidelines for the diagnosis and evaluation of therapy in Menière's disease. Otolaryngol Head Neck Surg 1995;113:181-5.

14 Nuti D, Nati C, Passali D. Treatment of benign paroxysmal positional vertigo: no need for postmaneuver restrictions. Otolaryngol Head Neck Surg $2000 ; 122: 440-4$. 